\title{
On nonlinear boundary value problems with impulses
}

Irena Rachunkova and Jan Tomeček 


\title{
ON NONLINEAR BOUNDARY VALUE PROBLEMS WITH IMPULSES
}

\author{
IRENA RACHUNKOVA AND JAN TOMEČEK \\ Department of Mathematics, Palacký University \\ Tomkova 40, 77900 Olomouc, Czech Republic \\ rachunko@risc.upol.cz, jan_tomecek@seznam.cz
}

[Received: November 2, 2001]

\begin{abstract}
This work deals with a nonlinear boundary value problem with impulses. Such a problem was investigated by many authors, see for example [1] or [2] and references therein. The main purpose of this paper is to prove the equivalence between impulsive problems and properly constructed boundary value problems without impulses. Using this equivalence we can transfer results known for boundary value problems of ordinary differential equations to boundary value problems of differential equations with impulses. Here, we use this approach and prove the lower and upper solutions method for nonlinear impulsive problems with impulsive functions having positive or negative derivatives.
\end{abstract}

Mathematical Subject Classification: 34A37, 34B15

Keywords: first order nonlinear ordinary differential equation, lower and upper functions, nonlinear boundary conditions, impulses

\section{Introduction}

Let us consider the interval $J=[a, b] \subset \mathrm{R}$, where $a=t_{0}<t_{1}<\ldots<t_{p}<t_{p+1}=b$. We will work with the Banach spaces $C(J)$ (the space of functions $x$ continuous on $J$ with the norm $\left.\|x\|_{C}=\max _{t \in J}|x(t)|\right), C^{1}(J)$ (the space of functions $x$ having continuous first derivatives on $J$ with the norm $\left.\|x\|_{C^{1}}=\|x\|_{C}+\left\|x^{\prime}\right\|_{C}\right), L(J)$ (the space of functions $y$ Lebesgue integrable on $J$ with the norm $\left.\|y\|_{1}=\int_{a}^{b}|y(t)| d t\right)$ and with the set $A C(J)$ (the set of functions absolutely continuous on $J$ ). We say that $f: J \times \mathrm{R} \rightarrow \mathrm{R}$ fulfils the Carathéodory conditions on $J \times \mathrm{R}$, if $f$ has the following properties: (i) for each $x \in \mathrm{R}$ the function $f(\cdot, x)$ is measurable on $J$; (ii) for almost each $t \in J$ the function $f(t, \cdot)$ is continuous on $\mathrm{R}$; (iii) for each compact set $K \subset \mathrm{R}$ the function $m_{K}(t)=\sup _{x \in K}|f(t, x)|$ is Lebesgue integrable on $J$. For the set of functions satisfying the Carathéodory conditions on $J \times \mathrm{R}$ we write $\operatorname{Car}(J \times \mathrm{R})$. For a subset $\Omega$ of a Banach space, $\operatorname{cl}(\Omega)$ and $\partial \Omega$ stand for the closure and the boundary of $\Omega$, respectively.

We will investigate the impulsive problem

$$
u^{\prime}(t)=f(t, u(t)) \quad \text { for a. e. } t \in\left(t_{j}, t_{j+1}\right), \quad j=0, \ldots, p,
$$




$$
\begin{gathered}
u\left(t_{j}+\right)=I_{j}\left(u\left(t_{j}\right)\right), \quad j=1, \ldots, p, \\
h(u(a), u(b))=0,
\end{gathered}
$$

where $f \in C a r(J \times \mathrm{R}), I_{j} \in C^{1}(\mathrm{R}), I_{j}^{\prime} \neq 0, j=1, \ldots, p$, and $h \in C\left(\mathrm{R}^{2}\right)$.

Together with problem (1.1) - (1.3) we will study the problem without impulses

$$
\begin{gathered}
x^{\prime}(t)=g(t, x(t)) \quad \text { for a. e. } t \in J, \\
h(x(a), w(x(b)))=0,
\end{gathered}
$$

where $g \in C a r(J \times \mathrm{R}), w \in C^{1}(\mathrm{R}), w^{\prime} \neq 0$, and $h \in C\left(\mathrm{R}^{2}\right)$.

We prove that problems (1.1) - (1.3) and (1.4), (1.5) are equivalent and by means of this we get the lower and upper functions method as well as the existence of solutions to problem (1.1) - (1.3). Let us note that the existence results for problem (1.1) (1.3) were proven also by E. Liz in [2], but by another approach and for increasing impulsive functions only. Here, we extend the lower and upper functions method and the existence results to the case of decreasing impulsive functions. Our proofs need no techniques or results from the theory of impulsive differential equations.

Definition 1. By $A C^{*}$ we mean a set of functions $u: J \rightarrow \mathrm{R}$, which are absolutely continuous on each $\left(t_{i}, t_{i+1}\right), i=0, \ldots, p, u\left(t_{j}\right)=u\left(t_{j}-\right), j=1, \ldots, p+1, u(a)=$ $u(a+)$. A function $u \in A C^{*}$ which satisfies conditions (1.1) - (1.3) is called a solution of problem (1.1) - (1.3). A function $x \in A C(J)$ which satisfies conditions (1.4), (1.5) is called a solution of problem (1.4), (1.5).

\section{Nonlinear boundary value problems without impulses}

To keep this paper self-contained, let us show the ideas of the lower and upper functions method for problem (1.4), (1.5).

Definition 2. A function $\alpha_{1} \in A C^{*}\left(\alpha_{2} \in A C^{*}\right)$ is called a lower (upper) function of problem (1.4), (1.5) provided the conditions

$$
\begin{gathered}
\left(\alpha_{i}^{\prime}(t)-g\left(t, \alpha_{i}(t)\right)\right)(-1)^{i} \geq 0 \quad \text { for a. e. } t \in\left(t_{j}, t_{j+1}\right), \quad j=0, \ldots, p, \\
\left(\alpha_{i}\left(t_{j}+\right)-\alpha_{i}\left(t_{j}\right)\right)(-1)^{i} \geq 0, \quad j=1, \ldots, p \\
h\left(\alpha_{i}(a), w\left(\alpha_{i}(b)\right)\right)(-1)^{i} \geq 0, \quad i=1,2
\end{gathered}
$$

are satisfied.

We will assume a certain relation between lower and upper functions.

First, let

$$
\alpha_{1}(t) \leq \alpha_{2}(t) \text { for each } t \in J
$$




$$
\begin{gathered}
\alpha(t, x)=\left\{\begin{array}{cll}
\alpha_{1}(t) & \text { if } & x<\alpha_{1}(t) \\
x & \text { if } & \alpha_{1}(t) \leq x \leq \alpha_{2}(t) \quad \text { for each } t \in J \text { and } x \in \mathrm{R}, \\
\alpha_{2}(t) & \text { if } & \alpha_{2}(t)<x
\end{array}\right. \\
\tilde{g}(t, x)=g(t, \alpha(t, x)), \quad g \in \operatorname{Car}(J \times \mathrm{R}) .
\end{gathered}
$$

Further, we assume that

$$
w \in C^{1}(\mathrm{R}), w^{\prime}>0 \text { on } \mathrm{R},
$$

$$
h \in C\left(\mathrm{R}^{2}\right), h \text { is nonincreasing in its second variable }
$$

and consider an auxiliary problem

$$
\begin{gathered}
x^{\prime}(t)=\tilde{g}(t, x(t)) \text { for a. e. } t \in J, \\
x(a)=\alpha(a, x(a)-h(x(a), w(x(b)))) .
\end{gathered}
$$

Proposition 1. Let us suppose (2.4) - (2.8) hold. Let $x$ be a solution of problem (2.9), (2.10) and let $\alpha_{1}, \alpha_{2}$ be lower and upper functions of problem (1.4), (1.5). Then

$$
\alpha_{1} \leq x \leq \alpha_{2} \text { on } J
$$

and consequently $x$ is a solution of (1.4), (1.5), as well.

Proof. Let us put

$$
z(t)=\alpha_{1}(t)-x(t) \text { for each } t \in J
$$

According to $(2.10)$ we have $x(a) \in\left[\alpha_{1}(a), \alpha_{2}(a)\right]$, which means that $z(a) \leq 0$. Suppose that there exists $q_{1} \in\left(a, t_{1}\right)$ such that

$$
z\left(q_{1}\right)>0 .
$$

Since $z$ is continuous on $\left[a, t_{1}\right)$, we can find $q_{0} \in\left[a, q_{1}\right)$ such that

$$
z\left(q_{0}\right)=0 \text { and } z>0 \text { on }\left(q_{0}, q_{1}\right] .
$$

In view of (2.1) we have

$$
z^{\prime}(t)=\alpha_{1}^{\prime}(t)-x^{\prime}(t) \leq g\left(t, \alpha_{1}(t)\right)-\tilde{g}(t, x(t))=0
$$

for a. e. $t \in\left(q_{0}, q_{1}\right]$. Therefore

$$
0 \geq \int_{q_{0}}^{q_{1}} z^{\prime}(t) d t=z\left(q_{1}\right)-z\left(q_{0}\right)=z\left(q_{1}\right),
$$

which contradicts (2.12). Thus we get

$$
z \leq 0 \text { on }\left[a, t_{1}\right] .
$$

By (2.2) and (2.14), the inequalities $\alpha_{1}\left(t_{1}+\right) \leq \alpha_{1}\left(t_{1}\right) \leq x\left(t_{1}\right)=x\left(t_{1}+\right)$ are true, and so $z\left(t_{1}+\right) \leq 0$.

Suppose that there exists $q_{1} \in\left(t_{1}, t_{2}\right)$ such that $(2.12)$ is true. Then we can find $q_{0} \in\left(t_{1}, q_{1}\right)$ such that $(2.13)$ is valid and we get a contradiction to (2.12) as before. In such a way we can argue at each interval $\left(t_{j}, t_{j+1}\right], j=1, \ldots, p$, and get $z \leq 0$ on $J$ which means that

$$
\alpha_{1} \leq x \text { on } J \text {. }
$$


The second inequality in (2.11) can be proved similarly putting $z=x-\alpha_{2}$ on $J$. Due to (2.11), we have

$$
x^{\prime}(t)=\tilde{g}(t, x(t))=g(t, x(t)) \text { for a. e. } t \in J .
$$

It remains to prove that $x$ fulfils (1.5). It is sufficient to show that

$$
\alpha_{1}(a) \leq x(a)-h(x(a), w(x(b))) \leq \alpha_{2}(a),
$$

because (2.10) implies (1.5) then.

Suppose on the contrary, that

$$
\alpha_{1}(a)>x(a)-h(x(a), w(x(b))) .
$$

Since (2.10) is valid, we get $x(a)=\alpha_{1}(a)$. Then, by (2.7) and (2.8), we have $0<h(x(a), w(x(b))) \leq h\left(\alpha_{1}(a), w\left(\alpha_{1}(b)\right)\right)$, which contradicts (2.3). The second inequality in (2.15) can be proved similarly. This completes the proof.

We can easily see that the following modification of Proposition 1 is true.

Proposition 2. The assertion of Proposition 1 remains valid if we replace the conditions (2.7) and (2.8) with

$$
w \in C^{1}(\mathrm{R}), w^{\prime}<0 \text { on } \mathrm{R}
$$

and

$$
h \in C\left(\mathrm{R}^{2}\right), h \text { is nondecreasing in its second variable. }
$$

Theorem 1. Let $\alpha_{1}$ and $\alpha_{2}$ be lower and upper functions of problem (1.4), (1.5). Further, suppose that $\alpha_{1} \leq \alpha_{2}$ on $J$ and that either $w^{\prime}>0$ and $h$ is nonincreasing in its second variable or $w^{\prime}<0$ and $h$ is nondecreasing in its second variable. Then there exists a solution $x$ of problem (1.4), (1.5) such that

$$
\alpha_{1} \leq x \leq \alpha_{2} \text { on } J .
$$

Proof. Consider the integral equation

$$
x(t)=\alpha(a, x(a)-h(x(a), w(x(b))))+\int_{a}^{t} \tilde{g}(s, x(s)) d s,
$$

which is equivalent to problem (2.9), (2.10). Further, define the set

$$
\Omega=\left\{x \in C(J):\|x\|_{C} \leq M\right\},
$$

where $M=\sup _{t \in J}\left|\alpha_{1}(t)\right|+\sup _{t \in J}\left|\alpha_{2}(t)\right|+\int_{a}^{b} \lambda(s) d s$ and $\lambda(t)=\sup \{|g(t, x)|: x \in$ $\left.\left[\alpha_{1}(t), \alpha_{2}(t)\right]\right\}$. Clearly $\Omega$ is a nonempty, convex, closed and bounded set in $C(J)$. We can check that the operator $T: \Omega \rightarrow C(J)$ given by

$$
(T x)(t)=\alpha(a, x(a)-h(x(a), w(x(b))))+\int_{a}^{t} \tilde{g}(s, x(s)) d s
$$


is continuous and that $\operatorname{cl}(T(\Omega))$ is a compact set in $C(J)$ and $T$ maps $\Omega$ to itself. Thus, according to the Schauder fixed point theorem, there is a point $x \in \Omega$ such that

$$
T x=x,
$$

which means that the function $x$ is a solution of (2.19) and consequently a solution of (2.9), (2.10). Propositions 1 and 2 imply that $x$ is a solution of (1.4), (1.5) and satisfies (2.18).

Now, suppose

$$
\alpha(t, x)=\left\{\begin{array}{cll}
\alpha_{2}(t) & \text { if } & x<\alpha_{2}(t) \leq \alpha_{1}(t) \text { for each } t \in J, \\
x & \text { if } & \alpha_{2}(t) \leq x \leq \alpha_{1}(t) \quad \text { for each } t \in J \text { and } x \in \mathrm{R} \\
\alpha_{1}(t) & \text { if } & \alpha_{1}(t)<x
\end{array}\right.
$$

and consider an auxiliary problem $(2.9)$,

$$
x(b)=\alpha(b, x(b)+h(x(a), w(x(b)))) .
$$

Proposition 3. Let conditions (2.20), (2.21), (2.6) and

$$
h \in C\left(\mathrm{R}^{2}\right), h \text { is nondecreasing in its first variable, } w \in C^{1}(\mathrm{R})
$$

be satisfied. Let $x$ be a solution of problem (2.9), (2.22) and let $\alpha_{1}, \alpha_{2}$ be lower and upper functions of problem (1.4), (1.5). Then

$$
\alpha_{2} \leq x \leq \alpha_{1} \text { on } J
$$

and consequently $x$ is a solution of (1.4), (1.5), as well.

Proof. This proof is similar to that of Proposition 1. We put

$$
z(t)=x(t)-\alpha_{1}(t) \text { for all } t \in J
$$

and prove $z(t) \leq 0$ for all $t \in J$. In view of $(2.22)$ we have $x(b) \in\left[\alpha_{2}(b), \alpha_{1}(b)\right]$, which means that $z(b) \leq 0$. Suppose that there is $q_{0} \in\left(t_{p}, b\right)$ such that

$$
z\left(q_{0}\right)>0 .
$$

Since $z$ is continuous on $\left(t_{p}, b\right]$, we can find $q_{1} \in\left(q_{0}, b\right]$ such that

$$
z\left(q_{1}\right)=0 \text { and } z>0 \text { on }\left[q_{0}, q_{1}\right) .
$$

In view of $(2.1)$ we have

$$
z^{\prime}(t)=x^{\prime}(t)-\alpha_{1}^{\prime}(t) \geq \tilde{g}(t, x(t))-g\left(t, \alpha_{1}(t)\right)=0 \text { for a. e. } t \in\left[q_{0}, q_{1}\right) .
$$

Therefore

$$
0 \leq \int_{q_{0}}^{q_{1}} z^{\prime}(t) d t=z\left(q_{1}\right)-z\left(q_{0}\right)=-z\left(q_{0}\right),
$$

which contradicts (2.25). Similarly, we can proceed to the initial point $a=t_{0}$. To prove the first inequality in $(2.24)$, we work with

$$
z(t)=\alpha_{2}(t)-x(t) \text { for each } t \in J .
$$

Due to (2.24), we have

$$
x^{\prime}(t)=\tilde{g}(t, x(t))=g(t, x(t)) \text { for a. e. } t \in J .
$$


Finally, we need to prove

$$
\alpha_{2}(b) \leq x(b)+h(x(a), w(x(b))) \leq \alpha_{1}(b) .
$$

If $\alpha_{1}(b)<x(b)+h(x(a), w(x(b)))$, then $x(b)=\alpha_{1}(b)$ and

$$
0<h(x(a), w(x(b))) \leq h\left(\alpha_{1}(a), w\left(\alpha_{1}(b)\right)\right),
$$

which contradicts (2.3). For the first inequality in (2.27) we argue similarly.

Theorem 2. Let $\alpha_{1}$ and $\alpha_{2}$ be lower and upper functions of problem (1.4), (1.5). Further, suppose that $\alpha_{1} \geq \alpha_{2}$ on $J$ and that $h$ is nondecreasing in its first variable. Then there exists a solution $x$ of problem (1.4), (1.5) such that

$$
\alpha_{2} \leq x \leq \alpha_{1} \text { on } J .
$$

Proof. Starting with the integral equation

$$
x(t)=\alpha(b, x(b)+h(x(a), w(x(b))))+\int_{b}^{t} \tilde{g}(s, x(s)) d s
$$

and using Proposition 3 instead of Propositions 1, 2, we can argue as in the proof of Theorem 1.

\section{Relation between impulsive problem (1.1)-(1.3) and problem (1.4),}

\section{(1.5)}

Consider the impulsive functions $I_{j} \in C^{1}(\mathrm{R}), I_{j}^{\prime} \neq 0, j=1, \ldots, p$, and define functions $w_{i}: \mathrm{R} \rightarrow \mathrm{R}, i=0, \ldots, p$ :

$$
\begin{aligned}
& w_{0}=\mathrm{id}_{\mathrm{R}}, \\
& w_{1}=I_{1}, \\
& w_{2}=I_{2}\left(I_{1}\right)=I_{2}\left(w_{1}\right), \\
& \cdots \\
& w_{p-1}=I_{p-1}\left(w_{p-2}\right), \\
& w=w_{p}=I_{p}\left(w_{p-1}\right) .
\end{aligned}
$$

Further, having $f \in \operatorname{Car}(J \times \mathrm{R})$, define

$$
g(t, x)=\left\{\begin{array}{lll}
\frac{f\left(t, w_{0}(x)\right)}{w_{0}^{\prime}(x)} & \text { for } t \in\left[a, t_{1}\right] \\
\frac{f\left(t, w_{1}(x)\right)}{w_{1}^{\prime}(x)} & \text { for } t \in\left(t_{1}, t_{2}\right] \\
& \cdots & \\
\frac{f\left(t, w_{p}(x)\right)}{w_{p}^{\prime}(x)} & \text { for } & t \in\left(t_{p}, b\right]
\end{array} \quad \text { for } x \in \mathrm{R} .\right.
$$

Conversely, having $g \in \operatorname{Car}(J \times \mathrm{R})$, define 


$$
f(t, u)=\left\{\begin{array}{lll}
g\left(t, w_{0}^{-1}(u)\right) w_{0}^{\prime}\left(w_{0}^{-1}(u)\right) & \text { for } t \in\left[a, t_{1}\right] & \\
g\left(t, w_{1}^{-1}(u)\right) w_{1}^{\prime}\left(w_{1}^{-1}(u)\right) & \text { for } & t \in\left(t_{1}, t_{2}\right] \\
& \cdots & \\
g\left(t, w_{p}^{-1}(u)\right) w_{p}^{\prime}\left(w_{p}^{-1}(u)\right) & \text { for } \quad t \in\left(t_{p}, b\right]
\end{array} \text { for } u \in \mathrm{R} .\right.
$$

Theorem 3. a) Let $u$ be a solution of problem (1.1) - (1.3) and let $g$ be defined by (3.1), (3.2). Then a function $x$ given by

$$
x(t)=\left\{\begin{array}{lll}
w_{0}^{-1}(u(t)) & \text { for } t \in\left[a, t_{1}\right] \\
w_{1}^{-1}(u(t)) & \text { for } t \in\left(t_{1}, t_{2}\right] \\
& \cdots & \\
w_{p}^{-1}(u(t)) & \text { for } & t \in\left(t_{p}, b\right],
\end{array}\right.
$$

is a solution of problem (1.4), (1.5).

b) Let $x$ be a solution of problem (1.4), (1.5) and let $f$ be defined by (3.1), (3.3).

Then a function $u$ given by

$$
u(t)=\left\{\begin{array}{lll}
w_{0}(x(t)) & \text { for } t \in\left[a, t_{1}\right] \\
w_{1}(x(t)) & \text { for } t \in\left(t_{1}, t_{2}\right] \\
& \cdots & \\
w_{p}(x(t)) & \text { for } t \in\left(t_{p}, b\right]
\end{array}\right.
$$

is a solution of problem (1.1) - (1.3).

Proof. a) Let $u$ be a solution of problem (1.1) - (1.3). Then, in view of (3.4) and $(3.2)$

$$
x^{\prime}(t)=\frac{u^{\prime}(t)}{w_{i}^{\prime}(x(t))}=\frac{f\left(t, w_{i}(x(t))\right)}{w_{i}^{\prime}(x(t))}=g(t, x(t)) \text { for a. e. } t \in\left(t_{i}, t_{i+1}\right),
$$

$i=0, \ldots, p$. Since $u(a)=x(a)$ and $u(b)=w_{p}(x(b))=w(x(b))$, we have by (1.3), $h(x(a), w(x(b)))=0$.

Next, $x\left(t_{j}+\right)=w_{j}^{-1}\left(u\left(t_{j}+\right)\right)=w_{j}^{-1}\left(I_{j}\left(u\left(t_{j}\right)\right)\right)=w_{j-1}^{-1}\left(I_{j}^{-1}\left(I_{j}\left(u\left(t_{j}\right)\right)\right)\right)=w_{j-1}^{-1}\left(u\left(t_{j}\right)\right)=$ $x\left(t_{j}\right), j=1, \ldots, p$. This together with $u \in A C^{*}$ implies $x \in A C(J)$ and therefore $x$ is a solution of (1.4), (1.5).

b) Let $x$ be a solution of (1.4), (1.5). Then, by (3.5) and (3.3), $u^{\prime}(t)=w_{i}^{\prime}(x(t)) x^{\prime}(t)=$ $w_{i}^{\prime}(x(t)) g(t, x(t))=w_{i}^{\prime}\left(w_{i}^{-1}(u(t))\right) g\left(t, w_{i}^{-1}(u(t))\right)=f(t, u(t))$ for a. e. $t \in\left(t_{i}, t_{i+1}\right), i=$ $0, \ldots, p$.

We have $x(a)=u(a), w(x(b))=w_{p}(x(b))=u(b)$ and then $h(u(a), u(b))=0$. Finally, $u\left(t_{j}+\right)=w_{j}\left(x\left(t_{j}+\right)\right)=w_{j}\left(x\left(t_{j}\right)\right)=I_{j}\left(w_{j-1}\left(x\left(t_{j}\right)\right)\right)=I_{j}\left(u\left(t_{j}\right)\right), j=1, \ldots, p$.

Since $x \in A C(J)$, then, in view of $(3.5), w_{i}(x(t))$ are absolutely continuous on $\left(t_{i}, t_{i+1}\right), i=0, \ldots, p$ and $u\left(t_{j}-\right)=u\left(t_{j}\right), j=1, \ldots, p+1$. Therefore $u$ is a solution of (1.1) - (1.3). 


\section{Lower and upper functions method to problem (1.1)-(1.3)}

\subsection{Increasing impulsive functions}

Let us suppose

$$
I_{j} \in C^{1}(\mathrm{R}), I_{j}^{\prime}>0, \quad j=1, \ldots, p .
$$

Definition 3. A function $\sigma_{1} \in A C^{*}\left(\sigma_{2} \in A C^{*}\right)$ is called a lower (upper) function of problem (1.1) - (1.3), provided the conditions

$$
\begin{gathered}
\left(\sigma_{i}^{\prime}(t)-f\left(t, \sigma_{i}(t)\right)\right)(-1)^{i} \geq 0 \text { for a. e. } t \in\left(t_{j}, t_{j+1}\right), \quad j=0, \ldots, p, \\
\left(\sigma_{i}\left(t_{j}+\right)-I_{j}\left(\sigma_{i}\left(t_{j}\right)\right)\right)(-1)^{i} \geq 0, \quad j=1, \ldots, p, \\
h\left(\sigma_{i}(a), \sigma_{i}(b)\right)(-1)^{i} \geq 0, \quad i=1,2
\end{gathered}
$$

are satisfied.

Theorem 4. Suppose that (4.1) holds.

a) Let $\sigma_{1}$ and $\sigma_{2}$ be lower and upper functions of problem (1.1) - (1.3) and let $g$ be defined by (3.1), (3.2). Then functions $\alpha_{1}$ and $\alpha_{2}$ given by

$$
\alpha_{i}(t)=\left\{\begin{array}{ccc}
w_{0}^{-1}\left(\sigma_{i}(t)\right) & \text { for } t \in\left[a, t_{1}\right] \\
w_{1}^{-1}\left(\sigma_{i}(t)\right) & \text { for } t \in\left(t_{1}, t_{2}\right] \\
& \cdots & \\
w_{p}^{-1}\left(\sigma_{i}(t)\right) & \text { for } t \in\left(t_{p}, b\right] .
\end{array}\right.
$$

are lower and upper functions of problem (1.4), (1.5).

b) Let $\alpha_{1}$ and $\alpha_{2}$ be lower and upper functions of problem (1.4), (1.5) and let $f$ be defined by (3.1), (3.3).

Then functions $\sigma_{1}$ and $\sigma_{2}$ given by

$$
\sigma_{i}(t)=\left\{\begin{array}{ccc}
w_{0}\left(\alpha_{i}(t)\right) & \text { for } t \in\left[a, t_{1}\right] \\
w_{1}\left(\alpha_{i}(t)\right) & \text { for } t \in\left(t_{1}, t_{2}\right] \\
& \cdots & \\
w_{p}\left(\alpha_{i}(t)\right) & \text { for } t \in\left(t_{p}, b\right],
\end{array}\right.
$$

are lower and upper functions of problem (1.1) - (1.3).

Proof. We will prove the assertion for lower functions only. Choose arbitrary $j \in$ $\{1, \ldots, p\}$ and $i \in\{0, \ldots, p\}$. Let $\sigma_{1}$ be a lower function of (1.1) - (1.3).

Then

$$
\alpha_{1}^{\prime}(t)=\frac{\sigma_{1}^{\prime}(t)}{w_{i}^{\prime}\left(\alpha_{1}(t)\right)} \leq \frac{f\left(t, \sigma_{1}(t)\right)}{w_{i}^{\prime}\left(\alpha_{1}(t)\right)}=g\left(t, \alpha_{1}(t)\right) \text { for a. e. } t \in\left(t_{i}, t_{i+1}\right) .
$$

Since $\sigma_{1}(a)=\alpha_{1}(a)$ and $\sigma_{1}(b)=w_{p}\left(\alpha_{1}(b)\right)=w\left(\alpha_{1}(b)\right)$, we have

$$
h\left(\alpha_{1}(a), w\left(\alpha_{1}(b)\right)\right)=h\left(\sigma_{1}(a), \sigma_{1}(b)\right) \leq 0 .
$$

Further, $\alpha_{1}\left(t_{j}+\right)=w_{j}^{-1}\left(\sigma_{1}\left(t_{j}+\right)\right) \leq w_{j}^{-1}\left(I_{j}\left(\sigma_{1}\left(t_{j}\right)\right)\right)=w_{j-1}^{-1}\left(\sigma_{1}\left(t_{j}\right)\right)=\alpha_{1}\left(t_{j}\right)$.

Thus $\alpha_{1}$ is a lower function of (1.4), (1.5).

Now, let $\alpha_{1}$ be a lower function of (1.4), (1.5). Then $\sigma_{1}^{\prime}(t)=w_{i}^{\prime}\left(\alpha_{1}(t)\right) \alpha_{1}^{\prime}(t) \leq$ $w_{i}^{\prime}\left(\alpha_{1}(t)\right) g\left(t, \alpha_{1}(t)\right)=w_{i}^{\prime}\left(w_{i}^{-1}\left(\sigma_{1}(t)\right)\right) g\left(t, w_{i}^{-1}\left(\sigma_{1}(t)\right)\right)=f\left(t, \sigma_{1}(t)\right)$, for a. e. $t \in$ 
$\left(t_{i}, t_{i+1}\right)$

As before, we have

$$
h\left(\sigma_{1}(a), \sigma_{1}(b)\right)=h\left(\alpha_{1}(a), w\left(\alpha_{1}(b)\right)\right) \leq 0 .
$$

Finally, $\sigma_{1}\left(t_{j}+\right)=w_{j}\left(\alpha_{1}\left(t_{j}+\right)\right) \leq w_{j}\left(\alpha_{1}\left(t_{j}\right)\right)=I_{j}\left(w_{j-1}\left(\alpha_{1}\left(t_{j}\right)\right)\right)=I_{j}\left(\sigma_{1}\left(t_{j}\right)\right)$. So, $\sigma_{1}$ is a lower function of (1.1) - (1.3).

Theorem 5. Let $\sigma_{1}$ and $\sigma_{2}$ be lower and upper functions of problem (1.1) - (1.3) and let (4.1) be fulfilled. Further, let one of the following conditions be satisfied:

(1) $\sigma_{1} \leq \sigma_{2}$ on $J$ and $h$ is nonincreasing in its second variable;

(2) $\sigma_{2} \leq \sigma_{1}$ on $J$ and $h$ is nondecreasing in its first variable. $J$.

Then there exists a solution of problem (1.1) - (1.3) lying between $\sigma_{1}$ and $\sigma_{2}$ on

Proof. We will investigate only the case $\sigma_{1} \leq \sigma_{2}$ on $J$. The second case can be proved similarly. Consider problem (1.4), (1.5), where $g$ is defined by (3.1), (3.2). We can see that $f \in \operatorname{Car}(J \times \mathrm{R})$ implies $g \in \operatorname{Car}(J \times \mathrm{R})$, and that, according to (4.1), we get $w \in C^{1}(\mathrm{R}), w^{\prime}>0$. Theorem 4 implies that the functions $\alpha_{1}, \alpha_{2}$ given by (4.5) are lower and upper functions of (1.4), (1.5).

Since the functions $w_{i}, i=0, \ldots, p$, are increasing, it follows that $\alpha_{1} \leq \alpha_{2}$ on $J$ if and only if $\sigma_{1} \leq \sigma_{2}$ on $J$. Therefore, by Theorem 1, problem (1.4), (1.5) has a solution $x$ which satisfies (2.18). Thus, in view of Theorem 3, the function $u$ given by (3.5) is a solution of (1.1) - (1.3) and $\sigma_{1} \leq u \leq \sigma_{2}$ on $J$.

\subsection{Decreasing impulsive function}

Let us suppose

$$
I_{j} \in C^{1}(\mathrm{R}), I_{j}^{\prime}<0, \quad j=1, \ldots, p .
$$

Definition 4. A function $\sigma_{1} \in A C^{*}\left(\sigma_{2} \in A C^{*}\right)$ is called a generalized lower (upper) function of problem (1.1) - (1.3), provided the conditions

$$
\begin{gathered}
\left(\sigma_{i}^{\prime}(t)-f\left(t, \sigma_{i}(t)\right)\right)(-1)^{i+j} \geq 0 \quad \text { for a. e. } t \in\left(t_{j}, t_{j+1}\right), \quad j=0, \ldots, p, \\
\left(\sigma_{i}\left(t_{j}+\right)-I_{j}\left(\sigma_{i}\left(t_{j}\right)\right)\right)(-1)^{i+j} \geq 0, \quad j=1, \ldots, p, \\
h\left(\sigma_{i}(a), \sigma_{i}(b)\right)(-1)^{i} \geq 0, \quad i=1,2 .
\end{gathered}
$$

Theorem 6. Suppose that (4.7) is satisfied.

a) Let $\sigma_{1}$ and $\sigma_{2}$ be generalized lower and upper functions of problem (1.1) - (1.3) and let $g$ be defined by (3.1), (3.2). Then $\alpha_{1}, \alpha_{2}$ given by (4.5) are lower and upper functions of (1.4), (1.5).

b) Let $\alpha_{1}, \alpha_{2}$ be lower and upper functions of (1.4), (1.5) and let $f$ be defined by (3.1), (3.3). Then $\sigma_{1}, \sigma_{2}$ given by (4.6) are generalized lower and upper functions of problem (1.1) - (1.3).

Proof. We can use the arguments from the proof of Theorem 4 having in mind that conditions (3.1) and (4.7) imply that

$$
(-1)^{j} w_{j}^{\prime}>0, j=0, \ldots, p .
$$


We will prove the assertion a) for $\sigma_{2}$, only. So, choose arbitrary $j \in\{0, \ldots, p\}$ and consider a generalized upper function $\sigma_{2}$ of (1.1) - (1.3). Then

$$
\alpha_{2}^{\prime}(t)-g\left(t, \alpha_{2}(t)\right)=\frac{\sigma_{2}^{\prime}(t)}{w_{j}^{\prime}\left(\alpha_{2}(t)\right)}-\frac{f\left(t, \sigma_{2}(t)\right)}{w_{j}^{\prime}\left(\alpha_{2}(t)\right)}=\left(\sigma_{2}^{\prime}(t)-f\left(t, \sigma_{2}(t)\right)\right) \frac{(-1)^{j}}{\left|w_{j}^{\prime}\left(\alpha_{2}(t)\right)\right|} \geq 0
$$

for a. e. $t \in\left(t_{j}, t_{j+1}\right)$.

Further, choose $j \in\{1, \ldots, p\}$ and suppose that $j$ is odd. Then, by $(4.9), \sigma_{2}\left(t_{j}+\right) \leq$ $I_{j}\left(\sigma_{2}\left(t_{j}\right)\right)$, and in view of (4.11) we have

$$
w_{j}^{-1}\left(\sigma_{2}\left(t_{j}+\right)\right) \geq w_{j}^{-1}\left(I_{j}\left(\sigma_{2}\left(t_{j}\right)\right)\right) .
$$

Therefore, $\alpha_{2}\left(t_{j}+\right)=w_{j}^{-1}\left(\sigma_{2}\left(t_{j}+\right)\right) \geq w_{j}^{-1}\left(I_{j}\left(\sigma_{2}\left(t_{j}\right)\right)\right)=w_{j-1}^{-1}\left(\sigma_{2}\left(t_{j}\right)\right)=\alpha_{2}\left(t_{j}\right)$. Similarly for $j$ if it is even. As in the proof of Theorem 4, we get $h\left(\alpha_{2}(a), w\left(\alpha_{2}(b)\right)\right) \geq$ 0 .

Theorem 7. Let $\sigma_{1}$ and $\sigma_{2}$ be generalized lower and upper functions of problem (1.1) - (1.3) and let (4.7) be fulfilled. Further, let one of the following conditions be satisfied:

(1) $\left(\sigma_{1}-\sigma_{2}\right)(-1)^{i} \leq 0$ on $\left(t_{i}, t_{i+1}\right]$ for $i=0, \ldots, p$ and $h$ is nonincreasing (nondecreasing) in its second variable if $p$ is even (odd);

(2) $\left(\sigma_{1}-\sigma_{2}\right)(-1)^{i} \geq 0$ on $\left(t_{i}, t_{i+1}\right]$ for $i=0, \ldots, p$ and $h$ is nondecreasing in its first variable. $J$.

Then there exists a solution of problem (1.1) - (1.3) lying between $\sigma_{1}$ and $\sigma_{2}$ on

Proof. We follow the proof of Theorem 5 working with $(-1)^{i} w_{i}, i=0, \ldots, p$ and using Theorem 6 instead of Theorem 4 .

We can also study, instead of problem (1.4), (1.5), problem (1.4),

$$
h(w(x(a)), x(b))=0,
$$

where $g \in \operatorname{Car}(J \times \mathrm{R})$ is given by

$$
g(t, x)=\left\{\begin{array}{lll}
\frac{f\left(t, w_{0}(x)\right)}{w_{0}^{\prime}(x)} & \text { for } t \in\left(t_{p}, b\right] \\
\frac{f\left(t, w_{1}(x)\right)}{w_{1}^{\prime}(x)} & \text { for } t \in\left(t_{p-1}, t_{p}\right] \\
\frac{f\left(t, w_{p}(x)\right)}{w_{p}^{\prime}(x)} & \text { for } t \in\left[a, t_{1}\right]
\end{array} \quad \text { for } x \in \mathrm{R},\right.
$$

where

$$
\begin{aligned}
& w_{0}=\mathrm{id}_{\mathrm{R}}, \\
& w_{1}=I_{1}^{-1} \\
& w_{2}=I_{2}^{-1}\left(I_{1}^{-1}\right)=I_{2}^{-1}\left(w_{1}\right), \\
& \cdots \\
& w_{p-1}=I_{p-1}^{-1}\left(w_{p-2}\right), \\
& w=w_{p}=I_{p}^{-1}\left(w_{p-1}\right) .
\end{aligned}
$$


Working with problem (1.4), (4.12) we can prove the following modification of Theorem 7 .

Theorem 8. Let $\sigma_{1}$ and $\sigma_{2}$ be generalized lower and upper function of problem (1.1) (1.3) and let (4.7) be fulfilled. Further, let one of the following conditions be satisfied:

(1) $\left(\sigma_{1}-\sigma_{2}\right)(-1)^{p-i} \leq 0$ on $\left(t_{i}, t_{i+1}\right]$ for $i=0, \ldots, p$ and $h$ is nonincreasing in its second variable;

(2) $\left(\sigma_{1}-\sigma_{2}\right)(-1)^{p-i} \geq 0$ on $\left(t_{i}, t_{i+1}\right]$ for $i=0, \ldots, p$ and $h$ is nondecreasing (nonincreasing) in its first variable if $p$ is even (odd).

Then there exists a solution of problem (1.1) - (1.3) lying between $\sigma_{1}$ and $\sigma_{2}$ on $J$.

Acknowledgement. The support by Grant No. 201/01/1451 of the Grant Agency of the Czech Republic and by the Council of the Czech Government J14/98:153100011 is gratefully acknowledged.

\section{REFERENCES}

[1] Bainov D. D., Hristova S. G., Hu S. and Lakshmikantham V.: Periodic boundary value problems for systems of first order impulsive differential equations, Differential and Integral Equations, 2, (1989), 37-43.

[2] Liz, E.: Existence and approximation of solutions for impulsive first order problems with nonlinear boundary conditions, Nonlin. Anal., TMA 25, (1995), 1191-1198. 